\title{
Enfoque exploratorio multivariante para el análisis de estructuras temporales. Una aplicación a la evolución de la demanda turística en España
}

\section{ISABEl LANDALUCE CALVO}

Universidad de Burgos, Facultad de Ciencias Económicas y Empresariales, Plaza Infanta Doña Elena, s/n, 09001 Burgos, España.E-mail: iland@ubu.es

\section{RESUMEN}

En este trabajo se lleva a cabo un estudio exploratorio de la estacionalidad inherente a la demanda turística en España desde una óptica metodológica novedosa. Se analiza el indicador mensual de las entradas de turistas según comunidad autónoma de destino principal, durante los últimos 10 años, desagregado en siete ámbitos geográficos. La originalidad de esta investigación radica en la estructuración de esta información en una tabla múltiple. Una combinación de Análisis Factorial Múltiple y Métodos de Clasificación es la técnica seleccionada como la más idónea para alcanzar los objetivos planteados, a través de sus numerosas medidas numéricas y gráficas.

Palabras clave: Series temporales, tablas múltiples, análisis factorial, clasificación

\section{Multivariate Exploratory Approach for the Analysis of Temporal Structures. An Application to the Evolution of the Tourist Demand in Spain}

\begin{abstract}
This paper carried out an exploratory study of the inherent seasonality of tourism demand in Spain from a new methodological perspective. It analyses the monthly indicator of tourist arrivals by main autonomous community destination for the last 10 years, disaggregated in seven geographical areas. The originality of this research lies in structuring this information in a multiple table. A combination of the Multiple Factor Analysis and the Classification Methods is the technical selected as the most suitable to achieve the objectives through its many numerical and graphical measures.
\end{abstract}

Keywords: Time Series, Multiple Tables, Factor Analysis, Classification.

Clasificación JEL: C38

Artículo recibido en octubre de 2016 y aceptado en febrero de 2017

Artículo disponible en versión electrónica en la página www.revista-eea.net, ref. ə-35204 


\section{INTRODUCCIÓN Y OBJETIVOS}

El turismo en España constituye un sector crucial para nuestra economía. La industria turística se caracteriza por su solidez y su consolidada posición de liderazgo a nivel internacional, contando además con gran potencial de crecimiento futuro. No obstante, desarrolla su actividad en un entorno económico globalizado, de gran incertidumbre y en el que continuamente surgen nuevas tendencias y retos. Por ello, el Consejo Español de Turismo, en base a esta realidad competitiva y cambiante, acordó llevar a cabo un amplio proceso de revisión estratégica del sector, que se ha materializado en el Plan del Turismo Español Horizonte 2020 (Secretaría General de Turismo, 2007). En esta revisión se incide en el análisis de los flujos turísticos de nuestro país, destacando como seña de identidad su desagregación temporal a lo largo de los meses del año y, sobre todo, su concentración en los meses de verano. Este perfil estacional del turismo nacional es objeto de importantes esfuerzos por parte de distintos agentes que se centran en el diseño de políticas turísticas para distribuir la llegada de visitantes en un periodo más extenso del año.

En este contexto, hay que señalar que la comunidad científica ha prestado considerable atención al estudio del turismo, en general, y al perfil estacional de la demanda turística, en particular. En Wanhill (2011) se puede encontrar una selección de trabajos realizados en este campo, distinguidos como "best paper" en la revista Tourism Economics. Pero este es sólo un ejemplo, ya que son varias las revistas, incluidas en el Social Science Citation Index, que han surgido en torno a la Economía del Turismo, con destacadas colaboraciones de autores españoles, como reflejan Albacete y Fuentes (2009), entre las que podemos destacar, junto a la ya mencionada, Tourism Management, Annals of Tourism Research, International Journal of Comtemporary Hospitality Management o Journal of Travel Research.

En lo que respecta al componente que nos ocupa, la estacionalidad, y centrándonos en la literatura especializada en nuestro entorno más cercano (ámbito nacional), se pueden encontrar numerosas referencias. Aún siendo diversos los aspectos analizados en las mismas, relacionados con el conocimiento de este fenómeno, todas coinciden en que se trata de uno de los principales desequilibrios de los destinos turísticos con nocivas consecuencias en términos laborales, medioambientales y de eficiencia económica; de ahí la importancia de su medición. Se presenta únicamente una selección de estos trabajos con el objetivo, por un lado, de realizar un recorrido histórico a lo largo de los últimos 30 años y, por otro lado, de dejar constancia de la investigación sobre todos los aspectos señalados con diferentes enfoques metodológicos. Aguiló y Sastre (1984) propusieron la medición de la estacionalidad, en el caso de Baleares, a través de los coeficientes de variación y de Gini, entendida ésta como desigualdad distribucional de la demanda turística a lo largo del año, incidiendo en la relación 
de este fenómeno con el desempleo en la región. Ramón y Abellán (1995), con la misma metodología, amplían el análisis de la estacionalidad al territorio nacional. Muñiz (2001), por su parte, utiliza el método X-11 ARIMA para evidenciar las graves consecuencias de la estacionalidad, especialmente en algunas CCAA, y, a su vez, presenta el turismo social como una tipología desestacionalizadora. López y López $(2006,2007)$ recogen una amplia revisión de la literatura sobre este tema, en la que detallan las principales líneas de investigación; en ambos trabajos vuelven a recuperar la metodología de Gini para medir la concentración del mercado turístico, si bien, hay que señalar que el primero de ellos tiene la originalidad de estar enfocado desde la oferta turística. Bujosa y Roselló (2011) ofrecen una óptica original, analizando, a través de un modelo de elección discreta, el efecto estacional del cambio climático sobre la probabilidad de visitar un determinado destino turístico. Riera y otros (2011) se atreven a medir la eficiencia de una muestra de establecimientos de alojamiento turístico en Baleares, a partir de la aplicación del análisis envolvente de datos, explicando parte de las diferencias encontradas en base al patrón estacional de la actividad turística. El trabajo de Duro y Farré (2015), además de recoger referencias actualizadas de la literatura científica sobre el tema, es otro exponente más del uso de las medidas de desigualdad para el análisis descriptivo de la concentración temporal en la demanda turística española; si bien, complementa el uso de los tradicionales coeficientes de variación y de Gini con los índices de la familia de Theil, comprobando que la aplicación de cada uno de ellos puede generar resultados distintos.

El presente artículo tiene su encuadre en este contexto de análisis exploratorio del perfil estacional del fenómeno de la demanda turística en España. Más concretamente, en términos comparativos a nivel regional. No obstante, su interés se focaliza, en esencia, en aportar un novedoso enfoque metodológico, desde un punto de vista multivariante. La estrategia propuesta en este trabajo se basa en el análisis simultáneo de un conjunto de series temporales yuxtapuestas, estructuradas como una tabla de tres dimensiones (también denominada tabla múltiple). Y está integrada por la combinación de una técnica de análisis factorial exploratorio adaptada al tratamiento de datos susceptibles de ser estructurados en varios grupos de variables, Análisis Factorial Múltiple (AFM), y de una técnica de Análisis de Clasificación (AC). Ninguna de las técnicas mencionadas ha sido aplicada para el análisis de series temporales o, al menos, no se han encontrado referencias en la comunidad científica al respecto. Únicamente en Aluja y Morineau (1999-pp. 114-125) se menciona una aplicación del Análisis de Componentes Principales a las temperaturas observadas diariamente en una treintena de lugares diferentes de una cueva durante dos años, quedando la dimensión tiempo como objetivo secundario. El AFM sí ha sido utilizado para el análisis de datos dinámicos. En García-Lautre y Abascal (2003) y Abascal y otros (2004) se presentan sendos 
estudios de análisis evolutivos, con datos tan diversos como infraestructuras en carreteras y educación de las CCAA o datos electorales, respectivamente; no obstante, en estas aplicaciones el tiempo ha sido utilizado como criterio de estructuración de los grupos y no como unidad estadística como se plantea en este artículo. Por lo que se puede considerar que este trabajo abre una nueva línea de investigación.

Este original enfoque no pretende ser un sustituto del análisis visual tradicional, que siempre será útil en las investigaciones preliminares de series temporales, sino una herramienta complementaria. El tándem AFM+AC no va a proporcionar una simple imagen de la evolución del fenómeno analizado en el tiempo, sino una visión detallada y, sobre todo, cuantificada de los acontecimientos más importantes ocurridos durante el periodo (componente estacional, tendencia, ...) para los diferentes contextos considerados, facilitando su estudio comparativo.

El objetivo aquí perseguido es, por tanto, doble. Por un lado, desde el punto de vista metodológico, presentar una nueva estrategia metodológica para visualizar y medir las posibles diferencias y/o las posibles semejanzas existentes entre diversas trayectorias temporales analizadas. Y, por otro lado, desde el punto de vista empírico, evidenciar la idoneidad de la técnica propuesta a través del análisis de la evolución de la demanda turística en nuestro entorno, desagregada a nivel regional.

El artículo se estructura como sigue. Después de esta contextualización, en el apartado segundo se describen brevemente las técnicas que integran la propuesta metodológica, introduciendo la apropiada estructuración de los datos, la terminología y los instrumentos gráficos y numéricos asociados a las mismas. A continuación, en el tercer apartado, se presentan e interpretan los resultados más destacados del análisis del perfil estacional de la demanda turística en los territorios a los que se circunscribe el estudio, ilustrando, a su vez, la adecuación del método para los objetivos perseguidos. En el apartado cuarto se discuten algunos resultados y se exponen las principales conclusiones, dejando abiertas otras líneas de investigación futuras. Por último, se aportan las referencias bibliográficas utilizadas.

\section{LOS DATOS, SU ESTRUCTURACIÓN y NOTAS METODOLÓGICAS}

En este trabajo se propone un enfoque diferente para el estudio de la estacionalidad inherente a la demanda turística en España, desde este punto de vista exploratorio. La exploración debe constituir una etapa previa en toda investigación que conlleve un análisis de datos. Y en el caso de las series temporales resulta extraordinariamente conveniente ya que, en ocasiones, se descubren regularidades que sirven de fundamento para la predicción del 
comportamiento de los fenómenos, incluso antes de que se entienda la razón o causa que justifique esas regularidades. Además, disponer de medidas de relación (semejanzas y diferencias) entre los diferentes contextos, permitirá la aplicación de otras técnicas de análisis para posteriores estudios con mayores garantías de éxito.

La idoneidad de nuestra propuesta va a quedar ilustrada a través de una detallada aplicación empírica. Para ello, se ha seleccionado el indicador que mide las "entradas de turistas según comunidad autónoma de destino principal” (correspondiente a la Encuesta de Movimientos Turísticos en Fronteras, FRONTUR) durante los últimos 10 años (enero 2006 - septiembre 2015). El Instituto de Estudios Turísticos (IET) proporciona esta información desagregada en siete ámbitos geográficos: Andalucía, Baleares, Canarias, Cataluña, C. de Madrid, C. Valenciana y resto de CCAA. Se ha trabajado con datos mensuales correspondientes a un período lo suficientemente amplio como para quedar reflejados hitos importantes en la evolución económica reciente de nuestra economía (final de una larga etapa de crecimiento, depresión y recuperación incipiente). Por tanto, los datos de partida están constituidos por siete series temporales, lo que amplía el objetivo inicial del estudio del perfil estacional de la demanda turística a un análisis comparativo a nivel geográfico. Todo ello, conlleva a la posibilidad de estructurar la información en una tabla múltiple (Figura 1) con tres dimensiones: indicador turístico, observaciones temporales y espacios geográficos.

Figura 1

Estructura de la información. Tabla múltiple

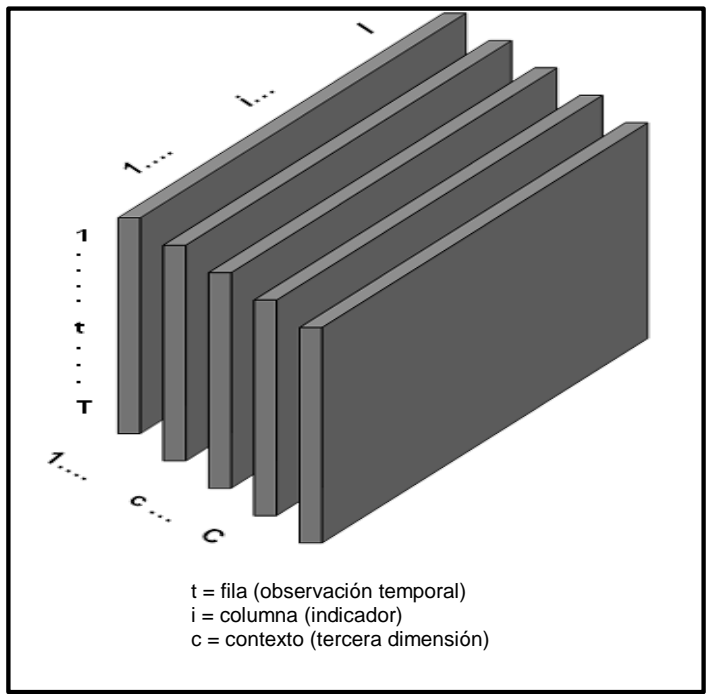

Fuente: Elaboración propia 
El tratamiento directo de las variables de esta naturaleza, dado su dinamismo, puede suponer una interpretación no exenta de dificultades. Si, además, el objetivo se amplía al estudio comparativo de estos fenómenos medidos en diferentes contextos (territoriales, como en nuestro caso) la complicación aumenta. Por ello, resulta conveniente realizar previamente un análisis descriptivo, con carácter exhaustivo, de las posibles realidades y/o relaciones que subyacen en el seno de este tipo de datos, habitualmente con gran riqueza de información.

Siguiendo la Figura 1, en el estudio aquí presentado la información consta de siete subtablas, $\mathrm{c}=1, \ldots, 7$, cada una de ellas asociada a uno de los ámbitos geográficos antes mencionados, respectivamente. Estas matrices de datos tienen en filas las observaciones mensuales, $\mathrm{t}=$ enero 2006,..., septiembre $2015 \mathrm{y}$, como característica destacable, una única columna relativa al fenómeno analizado (entradas de turistas). Hay que adelantar que, al ser grupos de una sola variable, el AFM proporciona ciertos resultados equivalentes a los obtenidos aplicando un ACP, Landaluce (1995). Sin embargo, el AFM ofrece una visión más detallada y, por ello, más completa de la estructura de los datos, desde un punto de vista tanto global (tabla única integrada por las siete series temporales), como parcial (subtablas integradas por cada serie temporal). Por este motivo, en la sección 4 se hará especial incidencia en los resultados propios de esta técnica de tabla múltiple.

El objetivo es obtener gráficamente y numéricamente un estudio detallado de las similitudes y de las diferencias que los siete contextos geográficos presentan en lo que respecta a la evolución del indicador medido y su perfil estacional en el periodo analizado. El análisis exploratorio desde la óptica de tabla múltiple y a través de la conjunción del AFM y del AC va a permitir contestar, entre otras, a las siguientes preguntas: ¿La trayectoria turística, desde el punto de vista de la demanda, ha sido similar en los siete territorios considerados? ¿Cuáles mantienen las mayores similitudes? ¿Y las mayores diferencias? ¿En qué unidades temporales se han producido?

Una vez detallada la estructuración de la información, a continuación se va a realizar una presentación de las técnicas a utilizar para su análisis, a través de unas breves y generales pinceladas, introduciendo la terminología propia de las mismas. Los instrumentos gráficos y los indicadores numéricos asociados, que ayudarán a su interpretación, serán presentados con mayor detalle en la aplicación empírica (sección 3), para un mejor seguimiento por los lectores no familiarizados con las técnicas factoriales y/o con el análisis de tablas múltiples. El desarrollo detallado de ambas metodologías, para aquellos que deseen profundizar en las mismas, pueden encontrarse, entre otros trabajos, en Abascal y Grande (2005), Escofier y Pagès (1986, 1992, 1994), Landaluce (1995) y Lebart y otros (2006). 
En términos generales, la metodología AFM se puede resumir en los siguientes puntos:

1. Es una técnica que permite el tratamiento simultáneo, como activas, de varias tablas de datos referidas al mismo conjunto de observaciones, pudiendo tener diferente número de variables y de distinta naturaleza (cualitativa o cuantitativa), aunque en el seno de cada una de ellas las variables deben ser de la misma naturaleza. Para las variables continuas, como en esta investigación, el AFM se comporta como un Análisis de Componentes Principales, ACP, (ponderando las variables).

2. A cada grupo de variables se asocia una nube de observaciones, que recibe el nombre de nube parcial. Así mismo, se define una nube global que resulta de la unión de todas las nubes parciales (previamente ponderadas), de cuyo análisis se obtendrán unos factores globales. Esta nube global tiene asociada una inercia que se denomina inercia total. En este método se descompone esta inercia total, de forma aditiva, en inercia intra e inercia inter.

3. Uno de los principales objetivos del AFM es examinar la existencia de estructuras comunes a todas, o aparte, de las nubes parciales, lo que se reflejaría en una inercia intra mínima o pequeña. Para ello, proporciona una representación superpuesta de estas nubes parciales proyectándolas sobre los ejes del análisis global de todos los grupos, como elementos ilustrativos

4. El AFM proporciona medidas globales de relación entre los grupos, basadas en el coeficiente de correlación vectorial entre matrices RV de Y. Escoufier (Robert y Escoufier, 1976).

Todos estos aspectos, a nivel práctico y de facilidad de interpretación, se concretan en dos etapas:

- Etapa de inter-estructura: análisis de la nube global, en el que cada grupo de variables es tratado como un ente y permite medir su relación.

- Etapa de intra-estructura: análisis comparativo de las nubes parciales en un espacio de referencia común, obtenido en la etapa anterior.

Por su parte, la metodología AC se puede resumir en los siguientes puntos:

1. Es un conjunto de métodos que obtiene grupos de observaciones semejantes de acuerdo con algún criterio. Estos objetos están descritos por cierto número de variables o indicadores y es necesario definir una medida de homogeneidad a partir de la cual se detectan los grupos.

2. Los métodos de clasificación difieren en la forma de obtención de los grupos: jerárquica ascendente, jerárquica descendente, no jerárquica y mixta. Los métodos jerárquicos construyen un árbol de clasificación (dendograma), que puede ser descendente o ascendente, siendo este 
último el más habitual y el que se va a aplicar en este trabajo. Utiliza el método de agregación de Ward, también llamado de varianza mínima.

3. Una vez elegida la partición (en función de la largura de las ramas), se obtienen, por un lado, los indicadores de la homogeneidad de las clases obtenidas: la inercia dentro de las clases (inercia intra-clase), indicador de la homogeneidad de los grupos y la inercia inter-clase que mide la diferencia entre los mismos. Por otro lado, un listado de las observaciones que pertenecen a cada clase, con gran utilidad en el análisis de la estacionalidad (como se comprobará en la aplicación empírica).

El análisis factorial y los métodos de clasificación se complementan y constituyen una idónea alternativa de exploración de datos (Aluja y Morineau, 1999). Por ello, en este trabajo se propone para el estudio detallado, y en términos comparativos, de varias series temporales, el uso conjunto de estas dos técnicas.

En la siguiente sección, a través de un estudio empírico con datos reales, se evidencia la idoneidad de esta propuesta. El programa estadístico utilizado ha sido Systéme Pour L'Analyse des Données (SPAD, versión 5.5). No obstante, señalar la disponibilidad de software libre, concretamente el paquete FactoMineR (Le y otros, 2008), creado en el seno del Laboratoire de Mathématiques Appliquées, Agrocampus Ouest, Rennes, Francia (se puede consultar más información en http://CRAN.R-project.org/package=FactoMineR).

\section{ESTRUCTURA TEMPORAL DE LA DEMANDA TURÍSTICA ESPAÑOLA}

En nuestra propuesta metodológica de análisis factorial exploratorio de la evolución temporal que a continuación presentamos, inicialmente realizaremos el estudio visual basado en el gráfico habitual de las series. Posteriormente, será completado con los resultados detallados del nuevo enfoque.

\subsection{Análisis previo}

El siguiente gráfico refleja la evolución del número de turistas de los siete territorios nacionales para los que se dispone de información desagregada.

Un estudio visual, en términos comparativos, de las series reflejadas en este gráfico permite alcanzar algunas conclusiones, desde un punto de vista general. No obstante, dado el carácter ilustrativo del mismo, no se va a profundizar en interpretaciones o valoraciones, ya que serán completadas posteriormente. Así, lo primero que se vislumbran son las conocidas diferencias de volumen de llegadas de turistas a las zonas seleccionadas; resultado esperado y lógico ya que se están comparando destinos turísticos muy distintos (turismo de sol y playa, turismo de interior,...) Esta diferente escala puede conllevar algunos 
riesgos en la interpretación de este gráfico que, sin otras herramientas métricas adicionales, siempre arrastra una carga de subjetividad. En este punto, señalar que en la metodología propuesta este riesgo no existe al quedar anulados estos desequilibrios, tipificando las unidades de medida de los indicadores analizados $\mathrm{y}$, por tanto, ponderando su influencia en los resultados. Una vez hecha esta puntualización, y continuando con la observación del gráfico, añadir que también permite concluir la existencia de un gran paralelismo en las trayectorias de evolución correspondientes a casi todas las zonas estudiadas, presentando, a priori, un cierto perfil estacional. Si bien, hay que destacar que las regiones de Madrid y de Canarias ofrecen un perfil peculiar. En el primer caso, este es menos marcado (menores diferencias entre temporadas altas y bajas) y en el segundo, el movimiento oscilatorio es contrario al del resto de trayectorias.

Figura 2

Evolución del número de turistas

(enero 2006-septiembre 2015)

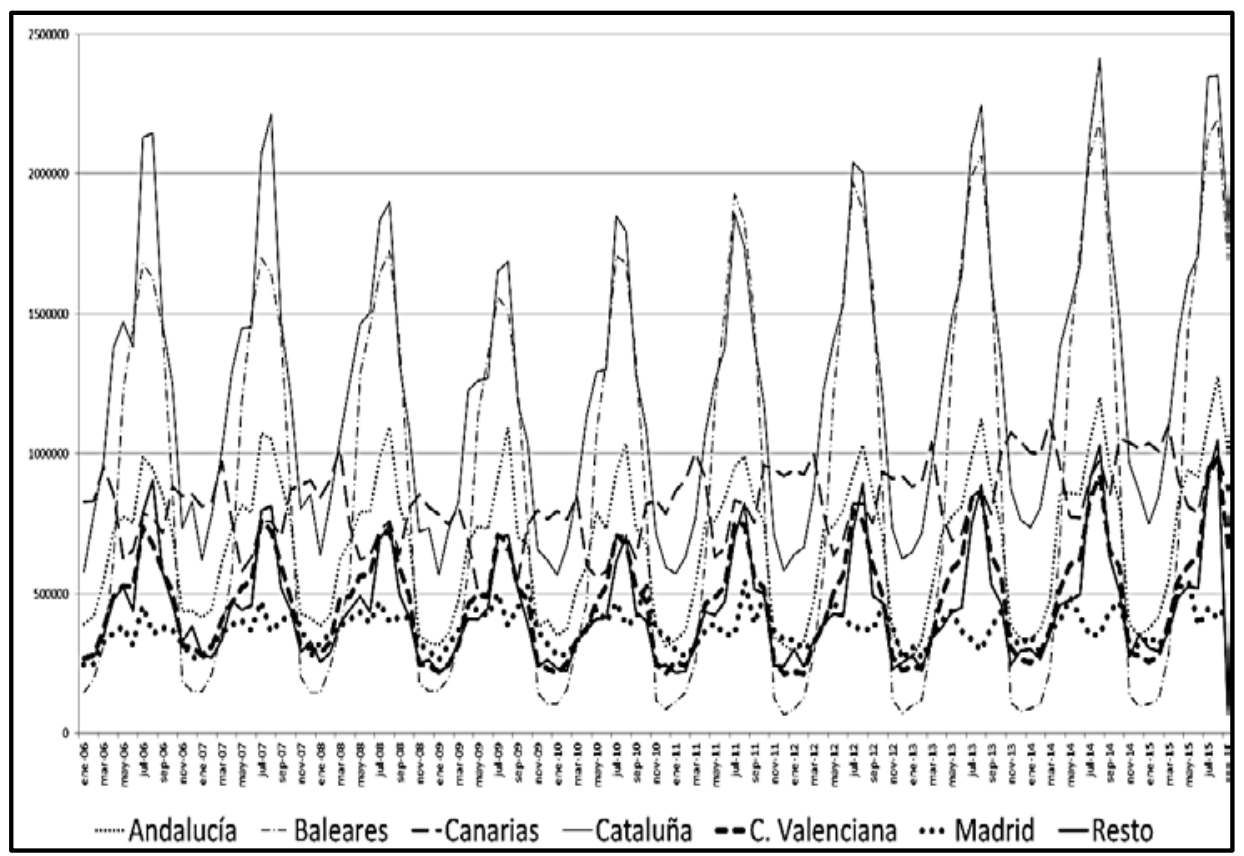

Fuente: Elaboración propia a partir de los datos del INE.

La nueva óptica que se propone en este trabajo proporciona una visión más detallada y, por ello, más completa de todas las posibles regularidades y de los patrones subyacentes en este tipo de información, desde un punto de vista tanto gráfico como métrico. 


\subsection{Análisis de la estacionalidad a través del AFM}

El AFM ofrece la doble visión antes citada, evitando el riesgo de subjetividad en la interpretación de sus gráficos, ya que los indicadores numéricos correspondientes permiten cuantificar las similitudes y las divergencias entre las series comparadas, matizando qué comunidades autónomas son las que presentan comportamientos con mayores similitudes, las que se separan en mayor medida de la trayectoria media y en qué momentos de tiempo se producen estos comportamientos. Además, realiza un análisis ponderado, en el que todos los contextos espaciales considerados tienen el mismo peso, independientemente de su "tamaño". Hay que adelantar que, al ser grupos de una sola variable, el AFM proporciona ciertos resultados equivalentes a los obtenidos aplicando un ACP (Landaluce, 1995), pero más completos y con gran utilidad para los objetivos aquí planteados.

Todo esto es posible al permitir el AFM un doble análisis de la tabla de datos: uno global, en el que se analiza la tabla como única (inter-estructura) y otro parcial (intra-estructura) en el que cada mes del periodo se proyecta desde cada uno de los siete puntos de vista (contextos geográficos) analizados.

Figura 3

Plano principal de los siete grupos (contextos geográficos)

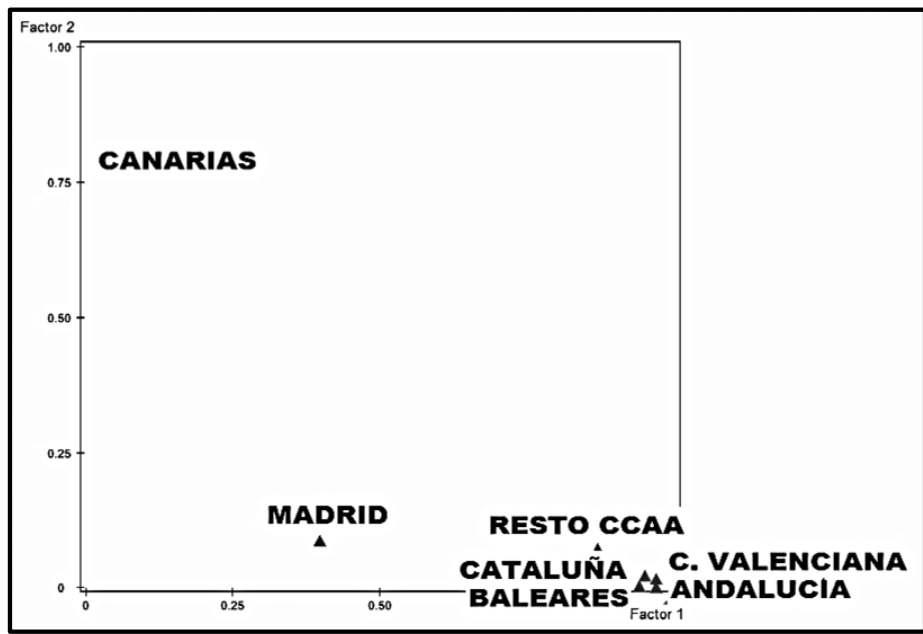

Fuente: Elaboración propia a partir de los resultados del programa SPAD.

Los primeros resultados que merecen ser destacados son aquellos que miden y reflejan un comportamiento global de los siete territorios:

- Plano principal de los grupos, contextos geográficos, (Figura 3): gráfico en el que cada grupo de variables aparece proyectado a través de un punto 
en un solo cuadrante. Se trata de una herramienta visual sencilla y muy clarificadora, ya que resume las regularidades y las particularidades de los grupos estudiados. La proximidad/distancia entre los puntos es reflejo de estas semejanzas y diferencias. Merece una atención especial, ya que para continuar el estudio propuesto, o para estudios posteriores, se puede tomar la decisión de eliminar, y por tanto, analizar alguno (o varios) de los grupos de manera aislada, al proyectarse a cierta distancia del resto.

En este análisis visual, se comprueba que hay dos núcleos geográficos con comportamientos peculiares: la Comunidad de Madrid y, de forma más notable, Canarias. Los otros cinco territorios considerados se proyectan muy próximos, lo que indica que tienen una realidad muy parecida: grandes similitudes en la evolución del indicador de demanda de turismo en el periodo analizado. Por su parte, la Comunidad de Madrid presenta ciertas similitudes (pero también diferencias) en su trayectoria temporal con estas zonas, mientras que la trayectoria de la Comunidad Canaria presenta destacables diferencias que habrá que analizar con detalle.

En esta investigación, y en base al objetivo nuclear de presentación de un nuevo enfoque metodológico, se ha decidido no eliminar ningún contexto espacial, hecho que permitirá poner de manifiesto la versatilidad de la técnica.

- Las medidas numéricas que completan la interpretación de este gráfico son la matriz de coeficientes $R V$ (Tabla 1) y las coordenadas y contribuciones de los grupos a los primeros factores del análisis global (Tabla 2).

Los coeficientes RV, medida de relación global entre grupos, corroboran los comportamientos más alejados del comportamiento medio tanto de la Comunidad de Canarias (con los menores valores), como de la Comunidad de Madrid (con coeficientes también muy bajos en general).

Tabla 1

Coeficientes RV de relación entre los grupos

\begin{tabular}{|l|c|c|c|c|c|c|c|}
\hline & $\mathbf{1}$ & $\mathbf{2}$ & $\mathbf{3}$ & $\mathbf{4}$ & $\mathbf{5}$ & $\mathbf{6}$ & $\mathbf{7}$ \\
\hline G1 - ANDALUCÍA & 1,00 & & & & & & \\
\hline G2 - BALEARES & 0,89 & 1,00 & & & & & \\
\hline G3 - CANARIAS & $\mathbf{0 , 1 1}$ & $\mathbf{0 , 1 8}$ & 1,00 & & & & \\
\hline G4 - CATALUÑ A & 0,92 & 0,89 & $\mathbf{0 , 0 6}$ & 1,00 & & & \\
\hline G5 - C. VALENCIANA & 0,95 & 0,92 & $\mathbf{0 , 0 7}$ & 0,96 & 1,00 & & \\
\hline G6 - MADRID & $\mathbf{0 , 3 6}$ & $\mathbf{0 , 2 8}$ & $\mathbf{0 , 0 9}$ & $\mathbf{0 , 2 6}$ & $\mathbf{0 , 3 1}$ & 1,00 & \\
\hline G7 - RESTO CCAA & 0,84 & 0,78 & $\mathbf{0 , 0 2}$ & 0,90 & 0,90 & $\mathbf{0 , 1 9}$ & 1,00 \\
\hline
\end{tabular}

Fuente: Elaboración propia a partir de los resultados del programa SPAD.

Respecto a los resultados reflejados en la Tabla 2, señalar que las mayores coordenadas y contribuciones sobre uno o varios de los ejes indican los 
grupos que han determinado en mayor medida dichos factores. La proximidad/distancia entre estos valores para varios grupos es reflejo de sus semejanzas/diferencias. Su observación permite confirmar la evolución similar del número de turistas experimentado en los últimos 10 años en Andalucía, Baleares, Cataluña, Comunidad Valenciana y Resto de CCAA (con destacables valores en el primer factor). Las coordenadas y las contribuciones de Canarias, en mayor medida, y de la Comunidad de Madrid corroboran su comportamiento peculiar. La evolución de la comunidad insular va a quedar reflejado en un segundo eje, presentando pocas similitudes con el resto de CCAA. Por su parte, la Comunidad de Madrid sí tiene similitudes con la trayectoria temporal reflejada en el primer factor, por lo que sus peculiaridades quedan relegadas a un tercer eje.

Tabla 2

Coordenadas y contribuciones de los grupos en los tres primeros factores del análisis global

\begin{tabular}{|c|c|c|c|c|c|c|}
\hline & \multicolumn{3}{|c|}{ Coordenadas } & \multicolumn{3}{|c|}{ Contribuciones } \\
\hline & F1 & F2 & F3 & F4 & F5 & F6 \\
\hline G1 - ANDALUCÍA & 0,97 & 0,00 & 0,00 & 18,5 & 0,10 & 0,00 \\
\hline G2 - BALEARES & 0,94 & 0,00 & 0,02 & 18,0 & 0,20 & 3,00 \\
\hline G3 - CANARIAS & 0,14 & 0,78 & 0,08 & 2,70 & 79,4 & 12,7 \\
\hline G4 - CATALUÑA & 0,95 & 0,02 & 0,01 & 18,1 & 2,30 & 1,00 \\
\hline G5 - C. VALENCIANA & 0,97 & 0,02 & 0,00 & 18,5 & 1,70 & 0,10 \\
\hline G6 - MADRID & 0,40 & 0,09 & 0,51 & 7,60 & 9,00 & 81,4 \\
\hline G7 - RESTO CCAA & 0,87 & 0,07 & 0,01 & 16,6 & 7,20 & 1,70 \\
\hline
\end{tabular}

Fuente: Elaboración propia a partir de los resultados del programa SPAD.

- El histograma de los valores propios del análisis de la tabla global y la razón entre la inercia inter y la inercia total (Tablas 3 y 4, respectivamente) son resultados que apoyan las conclusiones anteriores. Ambas medidas reflejan la existencia de un primer eje factorial preponderante que recoge casi un 75\% de la información inicial, por lo que los principales rasgos de la trayectoria temporal de la demanda turística, a nivel regional, quedan proyectados en él.

Tabla 3

Tres primeros valores propios del análisis global

\begin{tabular}{|c|c|c|}
\hline & Valor propio & Porcentaje de inercia \\
\hline $\mathbf{1}$ & 5,2400 & 74,86 \\
\hline $\mathbf{2}$ & 0,9790 & 13,99 \\
\hline $\mathbf{3}$ & 0,6305 & 9,010 \\
\hline
\end{tabular}

Fuente: Elaboración propia a partir de los resultados del programa SPAD. 
En lo que respecta al segundo, con una inercia del $14 \%$, en él quedan reflejadas las peculiaridades de la Comunidad de Canarias. El tercero (con apenas un 9\% de inercia) está asociado a algún rasgo presente en la comunidad madrileña. Estos mismos resultados se deducen de los valores alcanzados por la razón inercia inter/inercia total. Por ello, los esfuerzos interpretativos a partir de este momento se van a centrar en el plano principal, integrado por los dos primeros factores.

Tabla 4

Razón Inercia Inter / Inercia Total en los tres primeros ejes

\begin{tabular}{|c|c|c|}
\hline Factor $\mathbf{1}$ & Factor $\mathbf{2}$ & Factor $\mathbf{3}$ \\
\hline 0,75 & 0,14 & 0,09 \\
\hline
\end{tabular}

Fuente: Elaboración propia a partir de los resultados del programa SPAD.

Estos primeros resultados aún no señalan la existencia de un perfil estacional en la evolución de los turistas, únicamente ponen de manifiesto la existencia de similitudes y de diferencias en los comportamientos de las unidades espaciales analizadas. No obstante, el AFM proporciona más herramientas, tanto gráficas como numéricas, que completan y concretan los análisis anteriores y que, por tanto, permiten visualizar y medir los motivos de estas realidades encontradas. Entre ellas destacamos:

- El plano principal del análisis global (Figura 4), en el que aparecen proyectados los denominados "puntos medios". En este caso los puntos reflejan la posición media de las observaciones temporales (enero 2006 septiembre 2015) teniendo en cuenta conjuntamente las siete tablas analizadas. Así, se puede concluir que la parte positiva extrema del plano (cuadrantes 1 y 4) permite visualizar los meses de mayor afluencia de turistas: destacando julio y agosto. Además, se puede comprobar que esta realidad es más notable en los últimos años (crecimiento cronológico de las coordenadas correspondientes a los años 2013, 2014 y 2015), confirmando que en el sector turístico la senda del crecimiento económico se ha consolidado.

En la parte negativa (cuadrantes 2 y 3) quedan reflejados los meses en los que la afluencia de turistas es menor: noviembre, diciembre, enero, febrero y marzo, principalmente. Quedando situados en la zona central del plano los meses con una afluencia media: abril, mayo, junio, septiembre y octubre.

Por tanto, en este gráfico sí queda reflejada la existencia de un claro perfil estacional en la evolución de la demanda turística, como principal seña de identidad de la trayectoria correspondiente a la mayoría de las zonas estudiadas. También pone de manifiesto los meses de mayor y menor 
concentración de los flujos turísticos de nuestro país, según la desagregación espacial considerada. Ahora, resulta conveniente el análisis detallado, y desde un punto de vista comparativo, de las diferencias detectadas anteriormente entre las siete regiones.

Figura 4

Plano principal del AFM global.

Puntos medios: observaciones temporales ${ }^{1}$

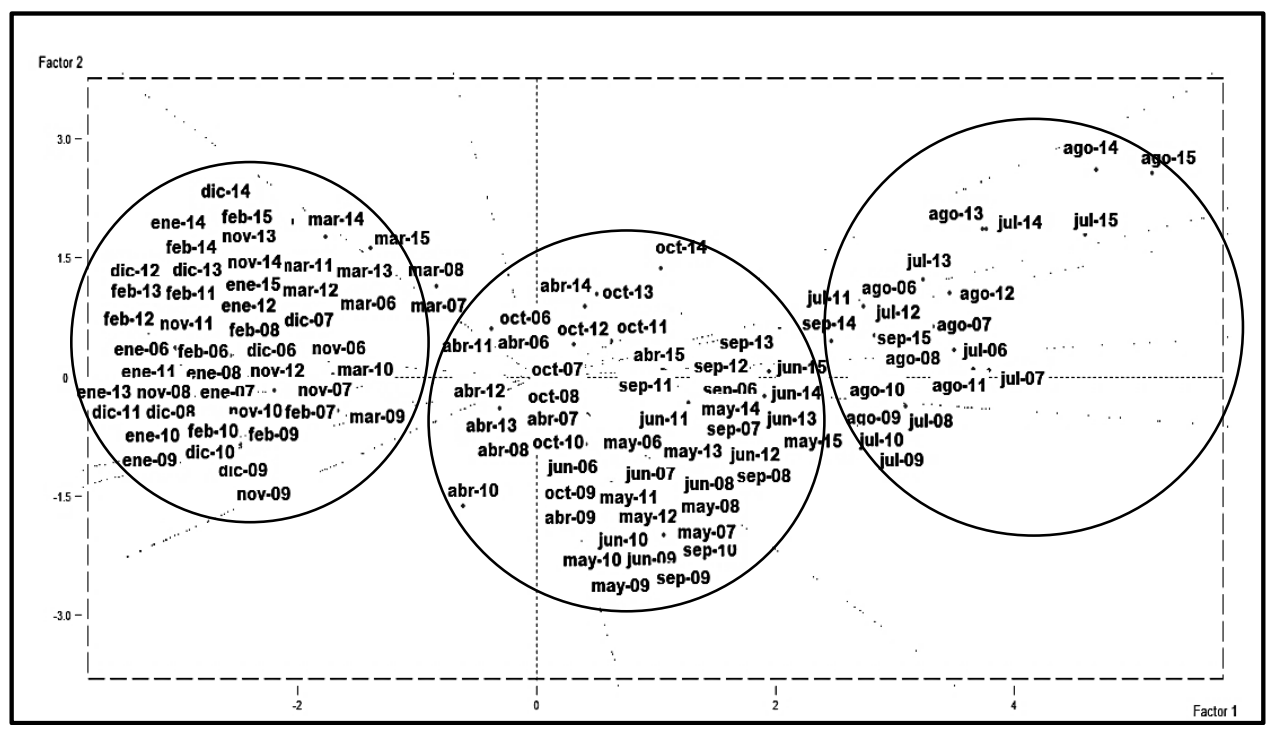

Fuente: Elaboración propia a partir de los resultados del programa SPAD.

- El plano principal de las observaciones parciales (Figura 5): en él aparecen proyectadas todas las observaciones temporales para todas y cada una de las observaciones espaciales analizadas y ayudará al estudio comparativo antes mencionado. Se trata de una representación gráfica conjunta de las trayectorias temporales de los siete contextos geográficos, constituyendo una nueva perspectiva gráfica del análisis comparativo de series temporales alternativa al gráfico habitual (Figura 2).

El hecho de que las trayectorias que refleja esta representación sean rectas está estrechamente relacionado con la filosofía del enfoque utilizado: análisis factorial exploratorio de una tabla múltiple integrada por grupos unidimensionales. Si algún lector está interesado en profundizar en este principio teórico del AFM puede consultar Landaluce (1995).

\footnotetext{
${ }^{1}$ La identificación de los puntos en el plano, aaa-XX, corresponde a las tres primeras letras del mes (aaa), a los dos últimos dígitos del año (XX).
} 
La observación de este plano permite comprobar cómo, de forma rápida, se visualizan claramente las regiones españolas con mayores similitudes (parecidos ángulos de separación de sus trayectorias respecto al eje horizontal). Se confirma que Andalucía, Baleares, Cataluña, C. Valenciana y resto de CCAA tienen claramente un comportamiento estacional (picos versus valles). Canarias, por su parte, no mantiene este comportamiento estacional y su proyección (más centrada en el primer eje, con la única coordenada negativa, y la mayor coordenada en el segundo) es reflejo de esta particularidad. Señalar que, por su parte, la Comunidad de Madrid, con una menor coordenada en el primer factor, y con un ángulo mayor respecto a las restantes cinco regiones, también mantiene un comportamiento menos estacional (ya se ha comentado que será el tercer eje el que refleje las particularidades de su trayectoria).

Figura 5

Plano principal de las observaciones parciales:

Trayectorias individuales de los siete contextos geográficos

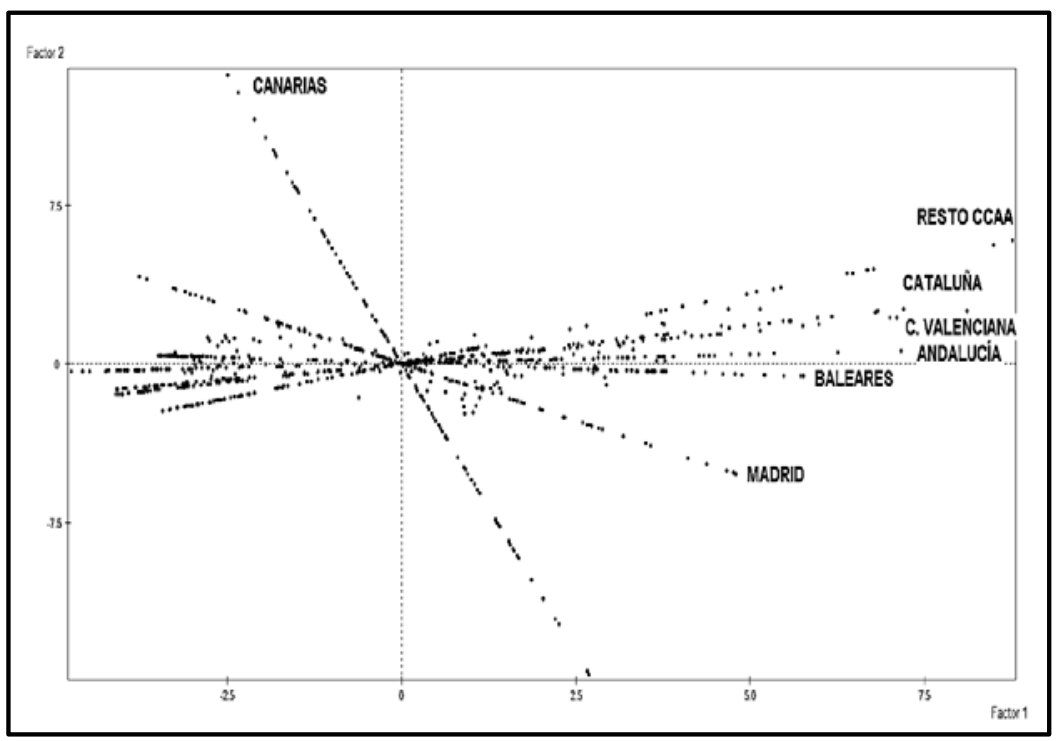

Fuente: Elaboración propia a partir de los resultados del programa SPAD.

La Comunidad de Canarias merece un estudio detallado que permita detectar los motivos de su particular trayectoria. Para ello, se puede utilizar un zoom del plano anterior, proyectando todos sus puntos parciales observaciones mensuales- (Figura 6). Este gráfico revela que el principal componente de los flujos de turistas en esta región insular es la tendencia (y no la estacionalidad como en el resto). Una tendencia que queda reflejada a 
lo largo del eje vertical (segundo factor), con claros repuntes en los últimos años de la serie (2014 y 2015, proyectados en el cuadrante 2). Los menores valores en el número de turistas llegados a las islas corresponden a los meses de los años más azotados por la reciente crisis económica (principalmente 2009 y 2010, proyectados en la parte negativa de este eje vertical, cuadrante 4). Otra peculiaridad que merece ser destacada: el hecho de que los repuntes en Canarias no se produzcan en los meses de mayor demanda para el resto de regiones, julio y agosto, sino en los meses en los que éstas reflejan menor flujo de turistas, enero, febrero, marzo, ...

Figura 6

Plano principal de las observaciones parciales:

Trayectoria individual de la Comunidad Canaria ${ }^{2}$

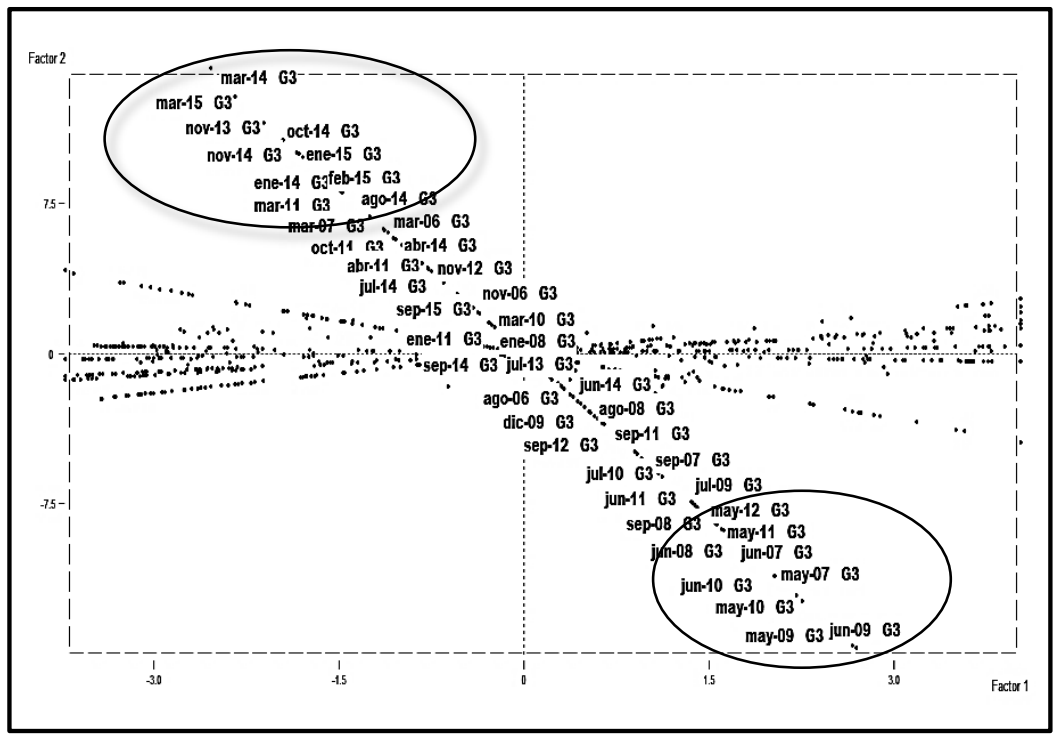

Fuente: Elaboración propia a partir de los resultados del programa SPAD.

La Comunidad de Madrid, por su parte, sí que presenta un cierto perfil estacional, aunque menos marcado que el resto, con mayores irregularidades. Por ello, merece ser estudiada también con cierto detalle, utilizando la misma herramienta que en el caso de Canarias. Para la búsqueda de sus peculiaridades, como ya se ha comentado, hay que recurrir al tercer factor (Figura 7). Por simplificación y, para ilustrar de forma clara la realidad de

\footnotetext{
${ }^{2}$ La identificación de los puntos en el plano, aaa-XX G3, corresponde a las tres primeras letras del mes (aaa), a los dos últimos dígitos del año (XX) y al grupo 3 (G3), con el que se asocia la variable "número de turistas llegados a la Comunidad de Canarias" en la estructuración como tabla múltiple de los datos iniciales.
} 
esta región, se han proyectado algunos puntos parciales, los de mayor coordenada, en el plano construido a partir del primer factor (eje horizontal) y tercer factor (eje vertical). Se puede comprobar que este último refleja los picos (parte negativa) y los valles (parte positiva) de la llegada de turistas a Madrid. En lo que respecta a los meses de menor afluencia, su comportamiento no presenta rasgos diferenciales. Sin embargo, en los meses de mayor afluencia se encuentra una de sus particularidades: en esta CCAA los repuntes no se producen siempre y únicamente en los meses de julio y agosto. Asimismo, hay que resaltar que el indicador turístico no se vio tan resentido en esta zona, como en otras, en los años caracterizados por una fuerte crisis económica en España.

Figura 7

Plano 1-3 de las observaciones parciales:

Detalle de la trayectoria individual de la Comunidad de Madrid $^{3}$

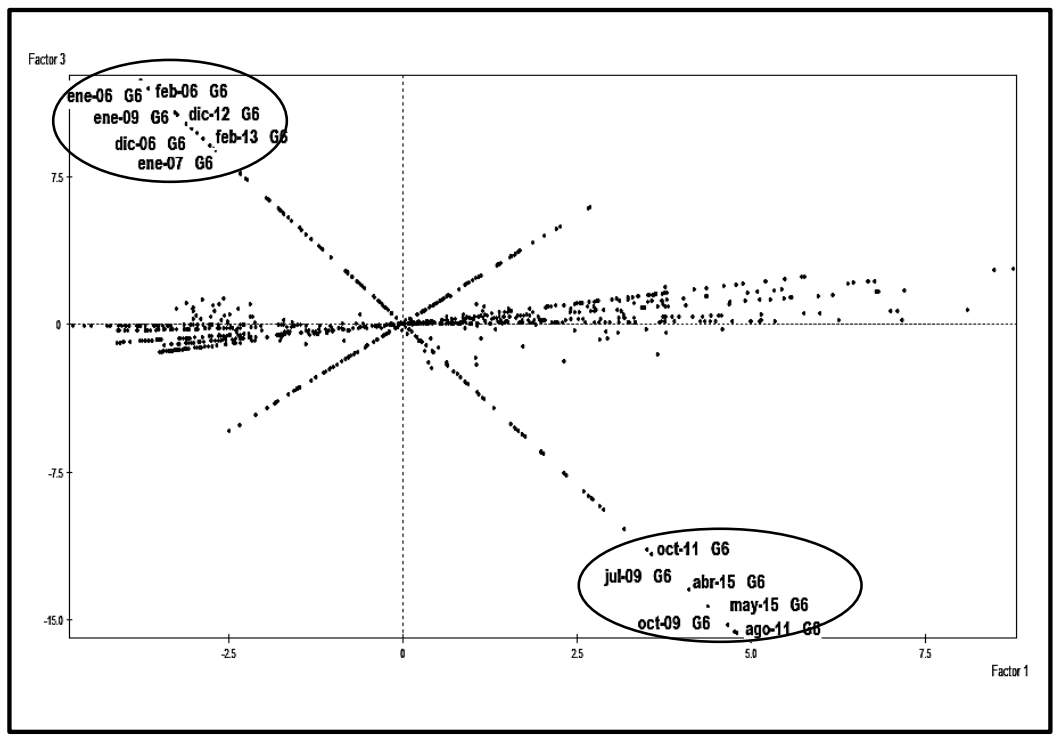

Fuente: Elaboración propia a partir de los resultados del programa SPAD.

Una herramienta numérica del AFM que avala las afirmaciones realizadas en los párrafos anteriores, sobre las características diferenciales de las trayectorias de las CCAA de Madrid y de Canarias, es la relación de los puntos parciales con las mayores inercias intra (Tabla 5). Esto es, las

\footnotetext{
${ }^{3}$ La identificación de los puntos en el plano, aaa-XX G3, corresponde a las tres primeras letras del mes (aaa), a los dos últimos dígitos del año (XX) y al grupo 6 (G6), con el que se asocia la variable "número de turistas llegados a la Comunidad de Madrid" en la estructuración como tabla múltiple de los datos iniciales.
} 
observaciones mensuales que se alejan, en mayor medida, del comportamiento medio y, por tanto, responsables de las diferencias que presentan algunos grupos (regiones, en este caso). Hay que recordar que el segundo factor está relacionado con el perfil turístico de Canarias (G3) y el tercero con el de Madrid (G6). Un rápido recorrido por las observaciones que se relacionan en las dos últimas columnas de la tabla permite comprobar su coincidencia con las unidades temporales señaladas en las conclusiones alcanzadas a partir de los gráficos correspondientes (Figuras 6 y 7).

Tabla 5

Individuos con mayor inercia intra en los tres primeros factores

\begin{tabular}{|c|c|c|}
\hline Factor 1 & Factor 2 & Factor 3 \\
\hline ago-15 G3 & jun-09 G3 & ago-11 G6 \\
ago-13 G6 & may-09 G3 & may-15 G6 \\
ago-14 G3 & mar-14 G3 & oct-10 G6 \\
jul-15 G3 & mar-15 G3 & oct-09 G6 \\
ago-14 G6 & sep-09 G3 & abr-15 G6 \\
jul-14 G3 & nov-13 G3 & ene-06 G6 \\
jul-14 G6 & may-10 G3 & jul-09 G6 \\
jul-13 G6 & may-07 G3 & feb-06 G6 \\
oct-10 G6 & jun-10 G3 & dic-12 G6 \\
jul-15 G6 & oct-14 G3 & ene-09 G6 \\
\hline
\end{tabular}

Fuente: Elaboración propia a partir de los resultados del programa SPAD.

\subsection{Análisis de la estacionalidad a través de AC}

Los métodos de clasificación, como ya se ha indicado en la sección anterior, son complementarios a los análisis factoriales y ayudan a completar y confirmar las conclusiones alcanzadas en la etapa anterior. En este caso, a partir de todos los factores obtenidos a través del AFM, AC confirmará la existencia de estacionalidad, aportando información adicional que completará su interpretación. Hay que tener en cuenta que este perfil de la demanda turística corresponde, de forma preponderante, al comportamiento medio de los contextos analizados, por lo que quedarán eclipsados aquellos grupos con rasgos propios. El método de clasificación elegido es el método jerárquico (sección 2), cuyo proceso queda esquemáticamente recogido en los siguientes resultados:

- Árbol de clasificación o dendograma (Figura 8): Proporciona visualmente suficiente información como para decidir el número adecuado de clases en las que dividir las observaciones mensuales (en base a la proximidad de sus correspondientes valores, número de turistas, en los territorios analizados). Siguiendo las indicaciones señaladas en la sección 2, son tres las clases resultantes en este estudio. El corte idóneo, dada la largura de las ramas, aparece indicado con una línea discontinua. Asimismo, se ha señalado el 
tamaño de cada una de las clases, con el número de observaciones que integra cada una.

Figura 8

Dendograma

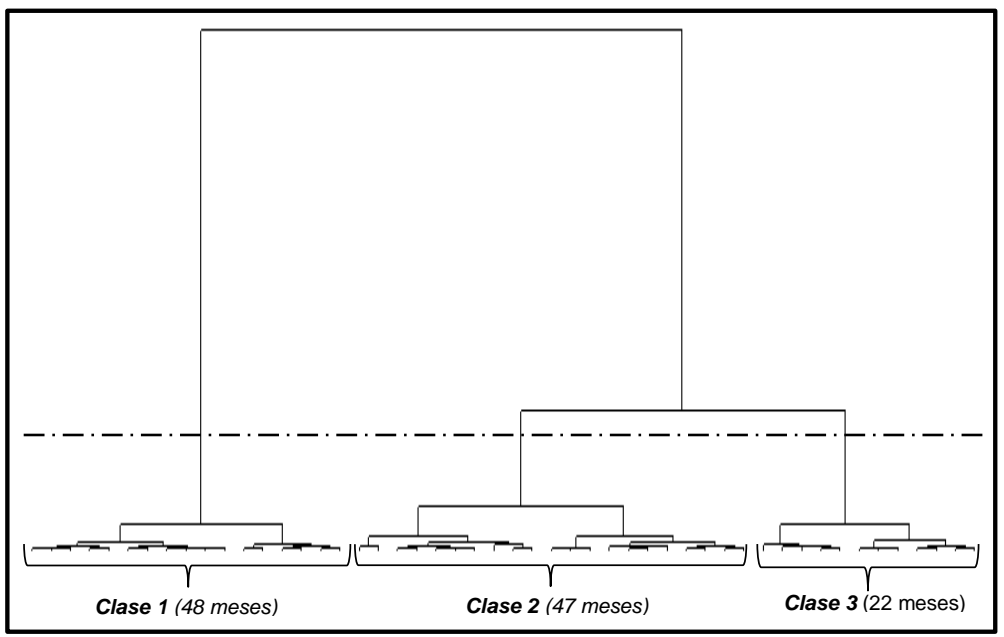

Fuente: Elaboración propia a partir de los resultados del programa SPAD.

- Inercia intra-clase e inter-clase (Tabla 6): Se trata de ayudas numéricas que permiten comprobar la adecuación de la clasificación seleccionada gráficamente. La primera, inercia dentro de las clases, es un indicador de la homogeneidad de cada uno de los grupos (por lo que resulta conveniente que alcance valores pequeños en todas las clases y parecidos entre sí) y la segunda mide las diferencias entre los mismos (por lo que un valor elevado es lo deseable).

Tabla 6

Descomposición de la inercia

\begin{tabular}{|l|l|}
\hline \multicolumn{2}{|c|}{ Inercias } \\
\hline Inter-Clases & 5,2286 \\
\hline Intra-Clase : & \\
- Clase 1 & 0,4677 \\
- Clase 2 & 0,8469 \\
- Clase 3 & 0,4179 \\
\hline Inercia Total & 6,9610 \\
\hline
\end{tabular}

Fuente: Elaboración propia a partir de los resultados del programa SPAD. 
En este caso, se comprueba que se han definido clases integradas por observaciones muy similares entre sí (inercia intra baja) y, a la vez, muy diferentes entre las que pertenecen a distintas (inercia inter alta). Esto es, la clasificación en este estudio proporciona una agrupación de los meses analizados en tres tipologías diferenciadas, que habrá que definir con la ayuda de resultados complementarios.

Tabla 7

Coordenadas y valores test de las clases en los dos primeros factores

\begin{tabular}{|c|cc|cc|}
\hline & \multicolumn{2}{|c|}{ Coordenadas } & \multicolumn{2}{c|}{ Valores-test } \\
& F1 & F2 & F1 & F2 \\
\hline Clase 1 & $-1,05$ & 0,33 & $-9,5$ & 3,0 \\
\hline Clase 2 & 0,38 & $-0,66$ & 3,4 & $-5,8$ \\
\hline Clase 3 & 1,49 & 0,70 & 7,7 & 3,6 \\
\hline
\end{tabular}

Fuente: Elaboración propia a partir de los resultados del programa SPAD.

- Coordenadas y valores-test de las clases sobre los ejes factoriales (Tabla 7 y Figura 9): Otra comprobación de la estabilidad de la clasificación elegida es la que proporciona la interpretación de las clases definidas, desde el punto de vista del fenómeno analizado. Para ello, existe la posibilidad de proyectar las tres clases obtenidas (a través de sus centros de gravedad) en el plano principal del AFM y medir su significatividad (valores-test superiores a $|2|$ ). Se comprueba que todas las clases tienen coordenadas significativas sobre los factores del plano principal analizado.

Figura 9

Plano Principal del AFM:

Proyección de los centros de gravedad de las clases

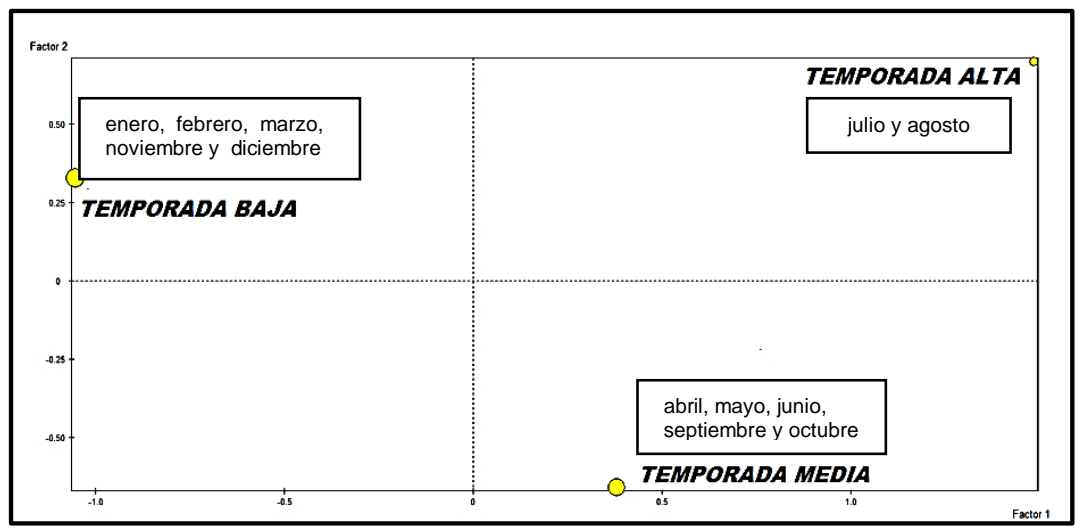

Fuente: Elaboración propia a partir de los resultados del programa SPAD. 
- Composición de las clases (Tabla 8): la relación de observaciones que integran cada una de las clases resulta de gran ayuda para confirmar la adecuación de la clasificación seleccionada y/o completar las interpretaciones que se han ido desgranando a partir de resultados anteriores. La visualización de esta tabla no solo corrobora la conclusión general relativa a la existencia de un claro perfil estacional en la demanda turística en España, sino que además, clarifica y agrupa los meses en los que se producen los diferentes hitos del perfil (lo que ha permitido completar la Figura 9).

La clasificación supone avanzar un paso más en la exploración de los datos, ya que proporciona una tipología de los meses según el nivel de concentración del flujo turístico, detectando la existencia de observaciones que se desvían de la trayectoria estacional e identificando las mismas. En este sentido, en la composición de la última clase se han señalado dos observaciones que merecen una atención especial por lo que representan: septiembre de 2014 y de 2015. Ambas pertenecen a la clase correspondiente a "temporada alta", integrada por los meses de julio y agosto de todos los años analizados. Este resultado induce a pensar que algo está cambiando en la trayectoria estacional "media" de las regiones españolas (con las excepciones ya comentadas): septiembre empieza a ser una época más de la denominada "temporada alta". Un resultado que habrá que seguir observando en la evolución futura y que los expertos deberán analizar en profundidad.

Tabla 8

Composición de las clases

\begin{tabular}{|l|l|l|l|l|l|l|l|l|l|l|l|l|}
\hline \multicolumn{7}{|c|}{ CLASE 1 } & \multicolumn{7}{c|}{ CLASE 2 } & \multicolumn{3}{c|}{ CLASE 3 } \\
\hline ene-06 & feb-06 & mar-06 & nov-06 & dic-06 & abr-06 & may-06 & jun-06 & sep-06 & oct-06 & jul-06 & ago-06 \\
ene-07 & feb-07 & mar-07 & nov-07 & dic-07 & abr-07 & may-07 & jun-07 & sep-07 & oct-07 & jul-07 & ago-07 \\
ene-08 & feb-08 & mar-08 & nov-08 & dic-08 & abr-08 & may-08 & jun-08 & sep-08 & oct-08 & jul-08 & ago-08 \\
ene-09 & feb-09 & mar-09 & nov-09 & dic-09 & abr-09 & may-09 & jun-09 & sep-09 & oct-09 & jul-09 & ago-09 \\
ene-10 & feb-10 & mar-10 & nov-10 & dic-10 & abr-10 & may-10 & jun-10 & sep-10 & oct-10 & jul-10 & ago-10 \\
ene-11 & feb-11 & mar-11 & nov-11 & dic-11 & abr-11 & may-11 & jun-11 & sep-11 & oct-11 & jul-11 & ago-11 \\
ene-12 & feb-12 & mar-12 & nov-12 & dic-12 & abr-12 & may-12 & jun-12 & sep-12 & oct-12 & jul-12 & ago-12 \\
ene-13 & feb-13 & mar-13 & nov-13 & dic-13 & abr-13 & may-13 & jun-13 & sep-13 & oct-13 & jul-13 & ago-13 \\
ene-14 & feb-14 & mar-14 & nov-14 & dic-14 & abr-14 & may-14 & jun-14 & & & jul-14 & ago-14 & sep-14 \\
ene-15 & feb-15 & mar-15 & & & abr-15 & may-15 & jun-15 & & & jul-15 & ago-15 & sep-15 \\
\hline
\end{tabular}

Fuente: Elaboración propia a partir de los resultados del programa SPAD.

- Caracterización de las clases (Tabla 9): el último resultado aportado por el método de clasificación que merece atención, dada la información que aporta, es el criterio que completa la descripción de las clases obtenidas. Este criterio, al trabajar con datos métricos, compara la media del indicador estudiado en el conjunto de observaciones (número medio de turistas en una zona determinada, correspondiente a la serie completa) con la media 
obtenida únicamente para los meses que integran cada clase. Así, una variable es característica de una clase si la media en ella resulta superior a la media general. Observando las medias reflejadas en la Tabla 9, se confirman, e incluso quedan clarificados, los rasgos peculiares de la Comunidad de Canarias: En la clase 1 presenta una media mayor que la media general; esto es, el número medio de turistas que llegan a esta región durante los meses catalogados de "temporada baja" para el resto de CCAA es mayor que el número medio de turistas llegados a lo largo de la serie analizada. Sin embargo, en la clase 2, catalogada como "temporada media", su número medio de turistas es menor que la media de la serie. En la clase 3, correspondiente a la "temporada alta" para el resto de regiones, el indicador de turismo de Canarias no resulta relevante.

Tabla 9

Caracterización de las clases

\begin{tabular}{|c|c|c|c|c|c|c|c|c|}
\hline \multicolumn{3}{|c|}{ Clase 1} & \multicolumn{3}{|c|}{ Clase 2} & \multicolumn{3}{|c|}{ Clase 3} \\
\hline$N^{0}$ turistas & $\begin{array}{c}\text { Media } \\
\text { clase }\end{array}$ & $\begin{array}{c}\text { Media } \\
\text { general }\end{array}$ & $N^{\circ}$ turistas & $\begin{array}{l}\text { Media } \\
\text { clase }\end{array}$ & $\begin{array}{c}\text { Media } \\
\text { general }\end{array}$ & $N^{\circ}$ turistas & $\begin{array}{l}\text { Media } \\
\text { clase }\end{array}$ & $\begin{array}{c}\text { Media } \\
\text { general }\end{array}$ \\
\hline Canarias & 909863 & 821740 & Madrid & 41816 & 376440 & Resto CCAA & 793519 & 450000 \\
\hline Madrid & 320636 & 376440 & Andalucía & 775781 & 669977 & Cataluña & 2005150 & 1235100 \\
\hline Resto CCAA & 283761 & 450000 & Baleares & 1131250 & 866888 & C. Valenciana & 764290 & 472593 \\
\hline Cataluña & 761376 & 1235100 & C. Valenciana & 529291 & 472593 & Andalucía & 1031240 & 669977 \\
\hline C. Valenciana & 283381 & 472593 & Canarias & 736752 & 821740 & Baleares & 1822840 & 866888 \\
\hline Baleares & 169892 & 866888 & & & & Madrid & 409058 & 376440 \\
\hline Andalucía & 400796 & 669977 & & & & & & \\
\hline
\end{tabular}

Fuente: Elaboración propia a partir de los resultados del programa SPAD.

El examen de todos estos resultados, como se ha comentado, avala las conclusiones alcanzadas en la etapa anterior, en lo que respecta al perfil estacional de la demanda turística. Pero, además, proporciona con mayor detalle los meses que integran cada "punto de inflexión" en las trayectorias temporales, poniendo de manifiesto la existencia de observaciones (meses) que se desvían, en algún momento determinado (año), del perfil medio. Todo ello, convierte al AC en una etapa de análisis complementaria a la anterior, no por ello, menos interesante, y siempre recomendable.

\section{CONCLUSIONES y OTRAS LÍNEAS DE INVESTIGACIÓN}

En este trabajo se ha realizado un estudio exploratorio exhaustivo de la estacionalidad de la demanda turística en España desde un nuevo enfoque, alternativo y complementario al habitual gráfico usado para la descripción de las trayectorias. La exhaustividad citada se debe a que se propone un análisis de las series temporales a través de una completa batería de instrumentos tanto visuales como métricos, que se suplementan entre sí. Además, permite comparar 
diferentes series, poniendo de manifiesto tanto sus semejanzas como sus diferencias. Por ello, el objetivo planteado en este artículo es doble, con aportaciones empíricas y metodológicas. No obstante, el núcleo fundamental de la aportación del mismo descansa sobre estas últimas, ya que la concentración temporal del flujo turístico en nuestro país ya ha sido analizada desde otras ópticas más tradicionales.

El indicador mensual de las entradas de turistas según comunidad autónoma de destino principal (Andalucía, Baleares, Canarias, Cataluña, C. de Madrid, C. Valenciana y resto de CCAA) durante los últimos 10 años ha sido la variable elegida para alcanzar el objetivo empírico. La originalidad de esta investigación radica en la estructuración de esta información en una tabla múltiple con tres dimensiones, consecuencia de la yuxtaposición de las series.

Una combinación de Análisis Factorial Múltiple (AFM) y Métodos de Clasificación (AC) ha sido la técnica seleccionada como idónea para visualizar y cuantificar la estructura temporal de la demanda turística en los siete destinos disponibles, en términos comparativos, a través de sus numerosas medidas numéricas y gráficas. Las ventajas que de esta metodología merecen ser destacadas se detallan a continuación:

- El distinto tamaño (en volumen de turistas) que presentan las unidades espaciales no supone ningún inconveniente para los objetivos planteados, ya que el AFM, al igual que las técnicas factoriales clásicas, pondera la influencia de las variables analizadas conjuntamente. En este caso, la ponderación también se extrapola a la nueva dimensión de grupos propia de esta técnica de análisis de tablas múltiples.

- El AFM proporciona planos factoriales en los que aparecen reflejadas desde una óptica distinta las series temporales. Por una parte, desde un punto de vista global, y en base a la estructuración en grupos de las series yuxtapuestas, permite ver en un solo gráfico todas las series temporales representadas a través de un solo punto cada una. Este gráfico ofrece una imagen simplificada de las series con mayores similitudes, las series peculiares, etc. Por otra parte, permite proyectar en un solo gráfico las series completas, obteniendo una imagen global de las unidades temporales en las que se producen tanto las semejanzas como las diferencias.

- En la interpretación de estos gráficos del AFM no existe el riesgo de caer en subjetividades, ya que a su vez proporciona una serie de indicadores numéricos que permiten cuantificar las similitudes y las divergencias entre las series comparadas. En este sentido, también proporciona resultados que permiten matizar en qué momentos de tiempo se producen y qué serie/s concreta/s es/son la/s responsable/s de estos comportamientos.

- El AC se presenta como una etapa de análisis complementaria a la anterior. No por ello, menos interesante, y siempre recomendable. También propor- 
ciona resultados gráficos y numéricos, que corroboran y, por tanto, avalan las conclusiones alcanzadas en la etapa anterior. El examen de estos resultados capacita al investigador para definir una agrupación de los meses, en un número determinado de clases, según el nivel de concentración del flujo turístico. Lo que conlleva una verificación del perfil estacional de la demanda turística ya detectado con el AFM, pero detallando los meses que integran cada "punto de inflexión” en la trayectoria temporal. Además, pone de manifiesto la posible existencia de observaciones (meses) que se desvían, en algún momento determinado (año), del perfil.

En lo que respecta al estudio de la estructura temporal de la demanda turística, que se ha utilizado como hilo conductor, señalar que sus resultados concretos han puesto de manifiesto todas estas ventajas. Entre las conclusiones alcanzadas merecen ser destacadas:

- La existencia de cinco regiones que, aún siendo de muy distinto tamaño, tienen grandes similitudes en el fenómeno concreto analizado: Andalucía, Baleares, Cataluña, C. Valenciana y resto de CCAA. Estas zonas presentan el mismo perfil estacional en la trayectoria mensual de la demanda turística correspondiente a los últimos 10 años. Este perfil se caracteriza por tener tres temporadas al año: la temporada de mayor demanda se concentra en los meses de julio y agosto; la temporada de menor demanda se produce en los tres primeros y los dos últimos meses del año, constituyendo el resto de los meses lo que se podría catalogar la temporada de demanda media. No obstante, hay que señalar que en los dos últimos años analizados, 2014 y 2015, se ha producido un fenómeno cuya continuidad habrá que seguir con detalle en el futuro: el repunte en el flujo turístico durante el mes de septiembre, hasta el punto de ser caracterizados como temporada alta.

- La Comunidad de Madrid también presenta un cierto perfil estacional, pero menos marcado que las citadas en el párrafo anterior, con mayores irregularidades. Por ello, ha sido estudiada por separado, detectando que en esta autonomía los repuntes no se producen siempre y únicamente en los meses de julio y agosto. Además, la afluencia de turistas no se vio tan resentida en esta zona en los años caracterizados por una fuerte crisis económica en España.

- La comunidad insular de Canarias ha merecido un estudio individualizado, al presentar una trayectoria diferente. Las peculiaridades detectadas al analizar los indicadores proporcionados por la metodología propuesta se pueden resumir en dos: por un lado, la demanda turística canaria no se caracteriza por un componente estacional, sino por una tendencia creciente y, por otro lado, la temporada alta se manifiesta en los primeros meses del año (y no en los meses de julio y agosto como en el resto de contextos geográficos analizados). 
Estas conclusiones evidencian que el análisis simultáneo de series temporales yuxtapuestas a través del tándem integrado por el Análisis Factorial Múltiple y el Análisis de Clasificación es un campo de investigación aplicada novedoso. Este trabajo, al plantearse como una presentación, con carácter prioritariamente ilustrativo, de la propuesta metodológica adolece de una serie de limitaciones que, a su vez, se erigen como futuras líneas de investigación que merecen ser exploradas, entre las que destacamos:

- Las reducidas dimensiones de la tabla a analizar, una variable por grupo, han representado una gran ventaja, ya que han permitido obtener representaciones gráficas y resultados numéricos de sencilla interpretación. El lector comprenderá que la potencialidad de esta técnica será más visible cuando los grupos a comparar sean de mayor dimensión, esto es, estén integrados por varias variables. Los objetivos del estudio se ampliarían a la búsqueda de una estructura de comportamiento interno en cada uno de los grupos (relación entre los indicadores en el seno de cada contexto) y estudio de las similitudes y diferencias entre dichos comportamientos. El AFM proporcionaría una visión más completa de las evoluciones de cada unidad espacial con trayectorias multidimensionales.

- En este mismo sentido, y en el ámbito del turismo, hay una realidad ineludible y es la existencia de diversos factores que se relacionan (pudiendo incluso ejercer alguna influencia) con su perfil estacional, por lo que sería conveniente ampliar el estudio con la incorporación de más variables.

- La dimensión relativa al número de grupos en los que estructurar la información inicial no tiene límite alguno, por lo que también podría resultar de interés medir este fenómeno a otros niveles espaciales (provincial, por ejemplo).

- La metodología propuesta en este trabajo se puede aplicar a datos categóricos. Como se ha dicho en la presentación, el AFM permite el estudio de variables nominales (e incluso de datos mixtos, siempre que las variables que integran cada grupo sean de la misma naturaleza). Por lo que, en la misma línea de los punto anteriores, podría completarse el análisis de los factores relacionados con la concentración de los flujos turísticos incorporando factores cualitativos y trabajar con tablas múltiples mixtas (Abascal y otros, 2006).

- Existen otras técnicas que permiten el análisis factorial de tablas estructuradas en grupos, con filosofía distinta, complementaria, a la del AFM. En este sentido, una posible aplicación podría ser comparar los resultados de varias de estas técnicas a partir del mismo conjunto de series temporales, comprobando las ventajas y los inconvenientes de cada una de ellas en estudios de naturaleza dinámica. Esta línea de trabajo sería similar a 
la seguida en el libro de Dazy el al. (1996), en el que los conjuntos de datos también son de carácter dinámico, pero el tiempo está asociado a la estructuración de las tablas (se comparan varias tablas, cada una de ellas referida a un momento de tiempo).

\section{REFERENCIAS BIBLIOGRÁFICAS}

ABASCAL E., GARCÍA-LAUTRE I. y LANDALUCE M.I. (2004). "Análisis de la evolución a través de encuestas. Trayectoria electoral de las comunidades autónomas españolas en el periodo 1977-2004". Metodología de Encuestas, Vol. 6, № 2, pp. 147-162.

ABASCAL E. y GRANDE I. (2005). Análisis de Encuestas. Madrid: ESIC.

ABASCAL, E., GARCÍA-LAUTRE y LANDALUCE, M. I. (2006). "Multiple factor analysis of mixed tables of metric and categorical data". Multiple Correspondence Analysis and related Methods. Edited by Jörg Blasius and Michael Greenacre. Chapman \& Hall (TAYLOR \&FRANCIS GROUP). Book Chapter 15, pp. 351-367.

AGUILÓ, E. (1984). "La medición de la estacionalidad del turismo: el caso de Baleares". Estudios Turísticos, núm. 81, pp. 79-88.

ALBACETE, C.A. y FUENTES-SÁEZ, M.M. (2009). "Difusión de la investigación española sobre turismo en revistas internacionales". XIV Congreso anual de AECIT, Gijón.

ALUJA, T y MORINEAU A. (1999). Aprender de los datos: El Análisis de Componentes Principales. Una aproximación desde el Data Mining. Barcelona: EUB.

BUJOSA, A. y ROSELLÓ, J. (2011). "Cambio climático y estacionalidad turística en España: Un análisis del turismo doméstico de costa". Estudios de Economía Aplicada, Vol. 29(3), pp. 863-880.

DAZY, F. y LE BARZIC, J.F. (1996). L'Analyse des Données Evolutives. Paris: Technip.

DURO, J.A y FARRÉ, F.X (2015). "Estacionalidad turística en las provincias españolas: Medición y Análisis". Cuadernos de Turismo, n³6, pp. 157-174.

ESCOFIER, B. y PAGES J. (1986). "Le traitement des variables qualitatives et tableaux mixtes par analyse factorielle multiple". Data Analysis and Informatics, IV(2), pp. 179191.

ESCOFIER, B. y PAGÈS, J. (1992). Análisis factoriales simples y múltiples. Objetivos, métodos e interpretación. Servicio editorial de la Universidad de País Vasco

ESCOFIER B. y PAGÈS J. (1994). "Multiple factor analysis (AFMULT package)“. Computational Statistics \& Data Analysis 18, pp.121-140. North-Holland.

EXCELTUR. Alianza para la excelencia turística. Informe Perspectivas Turísticas, № 58 , Octubre (2016).

GARCÍA-LAUTRE, I. y ABASCAL, E. (2003). "Una metodología para el estudio de la evolución de variables latentes. Análisis de las infraestructuras de carreteras de las comunidades autónomas (1975-2000)". Estadística Española, Vol. 45, № 153, pp. 193 a 210. 
LANDALUCE, M. I. (1995). Estudio de la estructura de gasto medio de las Comunidades Autónomas españolas. Una aplicación del Análisis Factorial Multiple. Tesis Doctoral. Universidad del País Vasco.

LANDALUCE, M. I., FERNÁNDEZ, K. y MODROÑO, J. I. (1999). "Reflexiones sobre el uso comparativo del Análisis Factorial Multiple (AFM) y de la Metodología Statis para el Análisis de Tablas Múltiples". Methodologica, Vol. 7, pp. 37-65.

LE, S., JOSSE, J. y HUSSON, F. (2008). "FactoMineR: An R Package for Multivariate Analysis". Journal of Statistical Software, 25(1). pp. 1-18.

LEBART, L., PIRON, M y MORINEAU, A. (2006). Statistique Exploratoire Multidimensionnelle. Visualisation et inférence en fouilles de données. Paris: Dunod. $4^{\mathrm{e}}$ édition

LOPEZ , J. M. y LOPEZ L. M. (2006). "La concentración estacional en las regiones españolas desde una perspectiva de la oferta turística". Revista de estudios regionales, $\mathrm{n}^{\circ} 77$, pp. 77-104.

LOPEZ , J. M. y LOPEZ L. M. (2007). "Variabilidad estacional del mercado turístico en Andalucía". Estudios y Perspectivas en Turismo, vol.16, pp. 150-172.

MUÑIZ, D. (2001). "El turismo social como tipología turística desestacionalizadora de la empresa hotelera." Cuadernos de CC.EE. y EE., № 41, pp. 93-109.

RAMÓN, A.B. y ABELLÁN, M.J. (1995) "Estacionalidad de la demanda turística en España". Papers de Turisme, 17, pp. 47-74.

RIERA, A. ; RIPOLL, A.M. y JUANEDA, C.N. (2011). "Efficiency and Seasonality in the Balearic Hospitality Industry". Estudios de Economía Aplicada, Vol. 29-3, pp. 845862.

ROBERT, P. y ESCOUFIER, Y. (1976). "A Unifying Tool for Linear Multivariate Statistical Methods: The RV-Coefficient”. Applied Statistics, 25(3), pp. 257-265.

SECRETARIA GENERAL DE TURISMO (2007). Plan del Turismo Español. Horizonte 2020. España : Ministerio de Industria, Comercio yTurismo.

SPAD v5.5 (2000). Systéme Portable d'Analyse des Donées Numeriques, CISIA, Montreuil, France.

WANHILL, S. (2011). "What Tourism Eonomist Do. Their Contribution to Unserstanding Tourism". Estudios de Economía Aplicada, Vol. 29(3), pp. 679-692. 
